\section{Mechanisms of sacubitril- valsartan benefit in HFrEF}

The PARADIGM-HF trial demonstrated that the angiotensin receptor-neprilysin inhibitor sacubitril-valsartan improves outcomes compared with the angiotensin-converting enzyme (ACE) inhibitor enalapril in patients with heart failure with reduced ejection fraction (HFrEF), but how the drug exerts its benefits remained unclear. Now, two studies presented at the ESC Congress 2019 reveal new details of the mechanisms, suggesting that sacubitril-valsartan induces reverse cardiac remodelling but has no effect on central aortic stiffness.

In the PARADIGM-HF trial, sacubitril-valsartan treatment resulted in a reduction of $\mathrm{N}$-terminal pro-B-type natriuretic peptide (NT-proBNP) levels and this reduction was associated with better prognosis. Given that NT-proBNP levels have been related to cardiac structure and function status, Januzzi and colleagues conducted the prospective PROVE-HF trial, which included 794 patients with HFrEF, to assess whether the reduction of NT-proBNP levels with sacubitril-valsartan treatment reflected improved cardiac performance. At 1 year, NT-proBNP levels had decreased by $37 \%$ from baseline, and this reduction weakly but significantly correlated with improvements in markers of cardiac volume and function, including left ventricular (LV) ejection fraction (with a $9.4 \%$ improvement from baseline), LV and left atrial volumes and the ratio of early diastolic filling to early diastolic annular velocity $\left(E / e^{\prime}\right)$. "The improvements in cardiac remodelling were comparable with, if not greater than, those seen with many other heart failure drugs," says Januzzi. Similar improvements were observed in prespecified subgroups, including patient populations not represented in PARADIGM-HF, such as patients not taking an ACE inhibitor or angiotensin-receptor blocker and those with new-onset HFrEF. The research team is now examining the effect of sacubitril-valsartan on symptoms and quality of life in the study population, and plans to study a broad range of mechanistic biomarkers.

The EVALUATE-HF trial, which included 464 patients with HFrEF, assessed the effect of sacubitril-valsartan on central aortic stiffness and cardiac remodelling compared with enalapril. At 12 weeks, no significant between-group differences were found in the change in central aortic stiffness, as assessed by aortic characteristic impedance. Patients receiving sacubitril-valsartan had greater reductions in LV and left atrial volumes and mitral $\mathrm{E} / \mathrm{e}^{\prime}$ ratio, but no significant differences were found for other secondary end points including LV ejection fraction.

"These results provide important information on how this drug exerts its benefit, suggesting that the benefit of sacubitril-valsartan in the PARADIGM-HF trial was related to improvement in cardiac structure and function," says Januzzi.

Irene Fernández-Ruiz

ORIGINAL ARTICLES Januzzi, J. L. et al. Association of change in N-terminal Pro-B-type natriuretic peptide following initiation of sacubitril-valsartan treatment with cardiac structure and function in patients with heart failure with reduced ejection fraction. JAMA https://doi.org/10.1001/jama.2019.12821 (2019) |Desai, A. S. et al. Effect of sacubitril-valsartan vs enalapril on aortic stiffness in patients with heart failure and reduced ejection fraction: a randomized clinical trial.JAMA https://doi.org/10.1001/ jama.2019.12843(2019)

\title{
Ticagrelor reduces ischaemic risk in patients with CAD and diabetes
}

Patients with stable coronary artery disease (CAD) and diabetes mellitus with or without previous percutaneous coronary intervention (PCI) are at high risk of ischaemic events. Two new trials presented at the ESC Congress 2019 now report that in these patients, ticagrelor added to aspirin significantly reduces the incidence of ischaemic events, but increases the risk of major bleeding compared with placebo plus aspirin.

Dual antiplatelet therapy (DAPT), a combination of aspirin plus a $\mathrm{P} 2 \mathrm{Y}_{12}$ receptor inhibitor, has been shown to improve outcomes for patients with acute coronary syndrome (ACS) or after coronary stenting. Patients with both CAD and diabetes are at high risk of ischaemic events, in part owing to increased platelet activation that is associated with insulin resistance. As such, aspirin treatment alone might not provide adequate protection for this high-risk population. The benefit of long-term DAPT on the risk of ischaemic events was explored in two patient populations with stable CAD and diabetes in the THEMIS and THEMIS-PCI trials.

In the randomized, doubleblinded THEMIS trial, 19,220 patients with stable CAD and diabetes with no history of myocardial infarction (MI) or stroke were randomly assigned to receive ticagrelor plus aspirin or placebo plus aspirin. The median duration of follow-up was 39.9 months. The incidence of cardiovascular death, MI or stroke (the primary efficacy outcome) was lower in the ticagrelor group than in the placebo group (7.7\% versus $8.5 \%$; HR $0.90,95 \%$ CI $0.81-0.99, P=0.04)$. However, the ticagrelor group had a higher incidence of major bleeding (as defined by the Thrombolysis in Myocardial Infarction (TIMI) criteria) than the placebo group (2.2\% versus $1.0 \%$; HR 2.32 , $95 \%$ CI 1.82-2.94, $P<0.001)$. Ticagrelor treatment was also associated with a slight excess of intracranial haemorrhage.

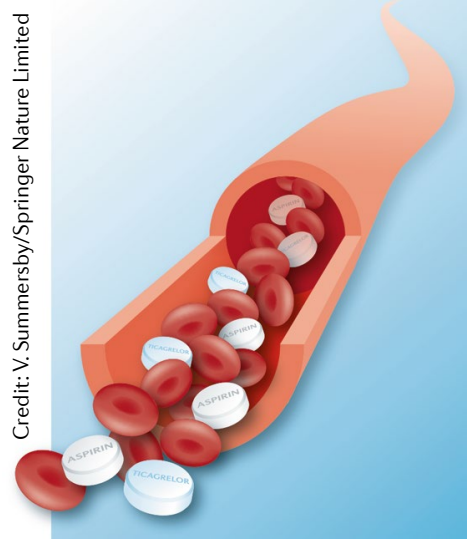

The THEMIS-PCI trial was a prespecified subgroup analysis of the THEMIS trial that was conducted to analyse the efficacy and safety of adding ticagrelor to aspirin in 11,154 patients who had previous PCI. Ticagrelor treatment was associated with fewer primary efficacy outcomes than placebo (7.3\% versus 8.6\%; HR 0.85, 95\% CI 0.74-0.97, $P=0.013)$. The incidence of TIMI major bleeding was significantly higher in the ticagrelor group, but the rates of fatal bleeding and intracranial haemorrhage were not significantly different between the groups.

"In carefully selected patients with diabetes and stable CAD who have a history of previous coronary stenting and have presumably tolerated DAPT in the past without bleeding complications, the combination of ticagrelor plus low-dose aspirin should be considered," explains Deepak Bhatt, who was involved in both trials. "These trial results provide a new option for high-risk patients with diabetes and extend the populations in which DAPT is already known to be beneficial, namely ACS, prior MI and recent coronary stenting."

Karina Huynh

ORIGINAL ARTICLES Steg, P. G. et al. Ticagrelor in patients with stable coronary disease and diabetes. N. Engl.J. Med. https://doi.org/10.1056/ NEJMoa1908077 (2019) |Bhatt, D. L. et al. Ticagrelor in patients with diabetes and stable coronary artery disease with a history of previous percutaneous coronary intervention (THEMIS-PCI) a phase 3, placebo-controlled, randomised trial. Lancet https://doi.org/10.1016/S01406736(19)31887-2 (2019) 